\title{
Variable Stars in the Sculptor Dwarf Galaxy
}

\author{
C. G. Goldsmith \\ York University, North York, Ontario, Canada
}

\section{Introduction}

This project was initiated in 1985 by James Nemec (University of Washington) and Nicholas Suntzeff (C.T.I.O.). The goal was to study the system of $\sim 600$ variable stars in the Sculptor dwarf galaxy. In 1987 the author became the recipient of the plate collection, which formed the basis for his $\mathrm{Ph} . \mathrm{D}$. dissertation. In this paper preliminary results are presented. Briefly, 612 stars were studied, of which 432 are van Agt (1978) stars and 180 are newly discovered variable stars. A total of 381 stars are confirmed variables. Most of these are RR Lyraes, but many anomalous Cepheids and some candidate eclipsing variables were also found. Several candidate double-mode RR Lyrae stars were also identified. The mean period of the $a b$-type RR Lyrae stars is $0.60 \pm 0.08$ day, and the mean period of the $c$-type stars is $0.35 \pm 0.03$ day, not unlike the mean periods of other nearby dwarf galaxies.

\section{Period-Amplitude Diagram}

Figure 1 shows the period-amplitude diagram for the RR Lyrae stars in Sculptor. The $a b$-types show the characteristic decrease in amplitude with increasing period, the longest period being $\sim 0.80$ day, characteristic of a very low metal abundance. Also, the $P-A_{B}$ relationship is significantly wider at a given amplitude than that for globular clusters (Sandage 1981), probably largely due to a range in metallicity for the stars. The slope of $P-A_{B}$ relation appears to be shallower than that for globular clusters. Several stars may also be misclassified in this diagram.

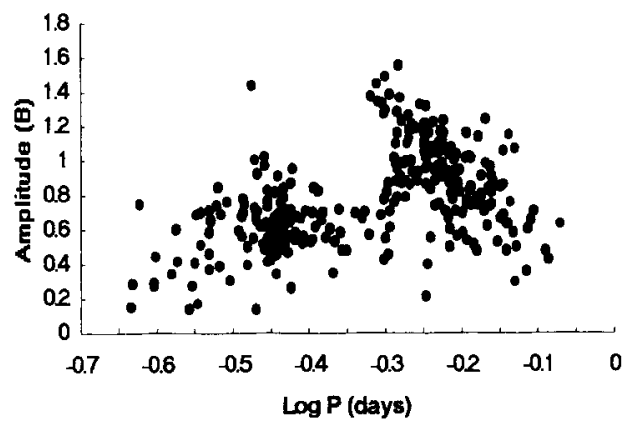

Figure 1. Period-amplitude diagram for the Sculptor variables.

\section{References:}

Sandage, A., 1981, Astrophys. J. 248, 168.

van Agt, S., 1978, Publ.David Dunlap Observatory, Vol.3 (number 7). 